\title{
The relationship between inflammatory mediator expression in the aqueous humor and secondary glaucoma incidence after silicone oil tamponade
}

\author{
ZHEN LIU ${ }^{1}$, GANG FU ${ }^{2}$ and AIHUA LIU ${ }^{3}$ \\ ${ }^{1}$ Department of Ophthalmology, The Second People's Hospital of Liaocheng, Linqing, Shandong 252601; \\ ${ }^{2}$ Department of Ophthalmology, The Second People's Hospital of Shaanxi Province, Xi'an, Shaanxi 710005; \\ ${ }^{3}$ Department of Ophthalmology, The Fourth Affiliated Hospital of Nanchang University, Nanchang, Jiangxi 330003, P.R. China
}

Received April 18, 2017; Accepted September 6, 2017

DOI: 10.3892/etm.2017.5269

\begin{abstract}
The expression of inflammatory mediators in the aqueous humor and the incidence of secondary glaucoma after vitreous body resection combined with silicone oil tamponade for patients with retinal detachment were inestigated, and the roles of IL-17, IL-6 and TNF- $\alpha$ in secondary glaucoma after silicone oil tamponade were analyzed. Fifty-eight cases of retinal detachment treated by vitrectomy combined with silicone oil tamponade were examined at the time of silicone oil removal surgery. All patients underwent unilateral surgery. Patients were divided into observation (19 cases with) and control (39 cases without) groups depending on development of secondary glaucoma. Prior to silicone oil removal, expression levels of IL-17, IL- 6 and TNF- $\alpha$ in the aqueous humor were examined. IL-17, IL- 6 and TNF- $\alpha$ levels in the aqueous humor in the observation group were $204.2 \pm 18.3,351.1 \pm 28.4$ and $850.0 \pm 51.7 \mathrm{pg} / \mathrm{ml}$, respectively, vs. $152.3 \pm 22.2,254.4 \pm 26.8$ and $625.6 \pm 61.2 \mathrm{pg} / \mathrm{ml}$, respectively in the control group $(\mathrm{P}<0.001)$. The expression of IL-17 was positively correlated with those of IL-6 and TNF- $\alpha$ ( $r=0.687,0.745$; P<0.001). IL-17, IL-6 and TNF- $\alpha$ presented good diagnostic values for glaucoma. The receiver operating characteristic (ROC) areas were 0.957 , 0.980 and 0.975 , respectively $(\mathrm{P}<0.001)$. The expression of inflammatory mediators such as IL-17, IL-6 and TNF- $\alpha$ in the aqueous humor of patients with secondary glaucoma after silicone oil tamponade significantly increased relative to patients without secondary glaucoma. The resultant inflammation may be involved in the development of secondary glaucoma.
\end{abstract}

Correspondence to: Dr Aihua Liu, Department of Ophthalmology, The Fourth Affiliated Hospital of Nanchang University, 133 Guangchangnan Road, Nanchang, Jiangxi 330003, P.R. China E-mail: draihualiu@hotmail.com

Key words: silicone oil tamponade, secondary glaucoma, interleukin-17, interleukin-6, tumor necrosis factor $\alpha$

\section{Introduction}

Patients with retinal detachment often require vitrectomies, and silicone oil is a commonly used intraocular filler (1). However, the long-term retention of silicone oil may interfere with intraocular energy metabolism and damage normal ocular tissues, leading to a variety of complications, including secondary glaucoma, corneal degeneration and silicone emulsion (2). Secondary glaucoma is a common disease with an incidence rate as high as $10-40 \%$ (3), and its mechanism of incidence and development are not yet fully understood. Currently, postoperative inflammation, leakage of silicone oil into the anterior chamber, silicone emulsion, and other factors are considered as primary candidates to be involved in the process (4). IL-17 is an inflammation mediator. It promotes the secretion of inflammatory cytokines, and IL- 6 and TNF- $\alpha$ are both effector molecules of IL-17 (5). The presernt study thus investigated the roles of IL-17, IL- 6 and TNF- $\alpha$ in secondary glaucoma after silicone oil tamponade.

\section{Patients and methods}

Inclusion and exclusion criteria. Inclusion criteria were as follows: i) retinal detachment patients who previously underwent vitrectomies combined with silicone oil tamponade currently undergoing silicone oil removal; ii) patients with unilateral eye lesions; iii) patients provided informed written consent; and iv) complete medical records for the patient were available. Exclusion criteria were as follows: i) patients with primary glaucoma, uveitis, and other autoimmune eye diseases prior to the silicone oil tamponade; ii) patients with high blood pressure, diabetes, systemic inflammation, or other systemic diseases; iii) patients with anterior chamber hyphema after silicone oil tamponade; iv) patients who had continuously used glucocorticoids for more than 2 weeks after the silicone oil tamponade; and v) patients who had systemic trauma.

Diagnostic criteria for secondary glaucoma. Secondary glaucoma was diagnosed in patients who had post-tamponade intraocular pressures (IOP) $\geq 24 \mathrm{mmHg}$, which was also 
Table I. Preoperative examination result comparison [n (\%)].

\begin{tabular}{lcccccc}
\hline Group (n) & $\begin{array}{c}\text { Silicone particles in } \\
\text { the anterior chamber }\end{array}$ & $\begin{array}{c}\text { Peripheral } \\
\text { anterior synechiae }\end{array}$ & Pupillary block & $\begin{array}{c}\text { Silicone } \\
\text { oil emulsion }\end{array}$ & $\begin{array}{c}\text { Artificial } \\
\text { intraocular lens }\end{array}$ & Aphakia \\
\hline Observation (19) & $10(52.6)$ & $4(21.1)$ & $2(10.5)$ & $6(31.6)$ & $2(10.5)$ & $3(15.8)$ \\
Control (39) & $17(43.6)$ & $5(12.8)$ & $2(5.1)$ & $11(28.2)$ & $2(5.1)$ & $4(10.3)$ \\
$\chi^{2}$ & 0.135 & $-{ }^{-a}$ & $-a$ & 0.002 & $-^{-a}$ & $-^{-a}$ \\
P-value & 0.713 & 0.456 & 0.591 & 0.966 & 0.591 & 0.673 \\
\hline
\end{tabular}

${ }^{\mathrm{a}}$ Exact probability test was used.

$\geq 10 \mathrm{mmHg}$ than their preoperative IOP. In addition, this increase in IOP had persisted $>6$ weeks.

General patient information. Fifty-eight patients treated between January 2015 and June 2016 who satisfied the inclusion and exclusion criteria were included in this study. Patients were divided into observation and control groups depending on the presence of secondary glaucoma. The observation group consisted of 19 patients (11 males and 8 females) with postoperative secondary glaucoma aged between 41 to 69 years (mean age, $51.6 \pm 8.3$ years). The time elapsed between silicon oil tamponade and silicon oil removal was between 3 to 36 months (average time, 10.8 \pm 7.1 months). These patients had elevated IOPs 2 days to 2.1 years after the surgery, with the maximal IOP values ranging from 32.1 to $51.4 \mathrm{mmHg}$ (average, $40.6 \pm 9.3 \mathrm{mmHg}$. The control group consisted of 39 patients (24 males and 15 females) without secondary glaucoma aged between 40 to 73 years (mean age, 51.1 18.1 years). The time between the silicon oil tamponade and the removal of silicon oil was between 3 and 32 months (average, 11.5 \pm 7.1 months). Maximal IOP values in this group were between 10.7 and $20.2 \mathrm{mmHg}$ (average, $15.3 \pm .1 \mathrm{mmHg}$ ). Other than IOP, no significant differences were observed in any of the general information criteria between the two groups $(P>0.05)$. This study was approved by the Ethics Committee of the Fourth Affiliated Hospital of Nanchang University.

Methods. Routine preoperative ultrasound biomicroscopy (UBM) and slit lamp examinations, as well as IOP measurements, were conducted prior to the removal of silicone oil. Before removal, aqueous humor samples were collected, placed in vials and stored at $-80^{\circ} \mathrm{C}$. Enzyme-linked immunosorbent assay (ELISA) was used to detect IL-17, IL-6 and TNF- $\alpha$ expression levels in aqueous humor samples. All ELISA kits were purchased from R\&D Systems (Minneapolis, MN, USA). All operations strictly followed the manufacturer's instructions. Optical density (OD) values at $450 \mathrm{~nm}$ were detected, and sample protein concentrations were determined using previously established protein concentration standard curves.

Statistical analysis. The SPSS 19.0 software (IBM Corp., Armonk, NY, USA) was used for data processing. Measurement data were expressed as mean \pm standard deviation. Comparisons between the groups were conducted using independent sample t-test. Correlation analysis was performed using Pearson correlation analysis. Count data were converted
Table II. Comparison of inflammatory mediator levels in the aqueous humor (mean $\pm \mathrm{SD}, \mathrm{pg} / \mathrm{ml}$ ).

\begin{tabular}{lccc}
\hline Group (n) & IL-17 & IL-6 & TNF- $\alpha$ \\
\hline Observation (19) & $204.2 \pm 18.3$ & $351.1 \pm 28.4$ & $850.0 \pm 51.7$ \\
Control (39) & $152.3 \pm 22.2$ & $254.4 \pm 26.8$ & $625.6 \pm 61.2$ \\
t-value & 8.805 & 12.651 & 13.750 \\
P-value & $<0.001$ & $<0.001$ & $<0.001$ \\
\hline
\end{tabular}

to proportions, and $\chi^{2}$ test or Fisher's exact probability test were used for comparisons. Receiver operating characteristic (ROC) curves were used to determine the diagnostic abilities of IL-17, IL-6 and TNF- $\alpha$ for secondary glaucoma, as well as to calculate the sensitivity, specificity and other indicators. $\mathrm{P}<0.05$ was considered to indicate a statistically significant difference.

\section{Results}

Comparisons of the preoperative examination results of the two groups. UBM examinations revealed that 27 of the 58 patients $(46.6 \%)$ had silicone particles in the anterior chamber, $9(15.5 \%)$ patients had peripheral anterior synechiae and 4 (6.9\%) patients had pupillary block. Slit lamp microscope examinations showed that 17 (29.3\%) patients had silicone oil emulsion, 4 (6.9\%) patients had artificial intraocular lens and 7 (12.1\%) patients had aphakia. The incidence of these complications in the observation and control groups were not significantly different $(\mathrm{P}>0.05$; Table $\mathrm{I})$.

Comparison of inflammatory mediator levels in the aqueous humor. The observation group had higher levels of IL-17 (204.2 \pm 18.3$)$, IL-6 (351.1 \pm 28.4$)$, and TNF- $\alpha(850.0 \pm 51.7)$ than those in the control group $(152.3 \pm 22.2,254.4 \pm 26.8$ and

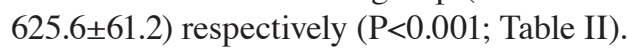

Correlation of IL-17 with IL-6 and TNF- $\alpha$. IL-17 levels showed positive correlations with IL-6 $(\mathrm{r}=0.697 ; \mathrm{P}<0.001)$ and TNF- $\alpha(r=0.745, \mathrm{P}<0.001)$ levels (Fig. 1$)$.

Diagnostic value of aqueous humoral IL-17, IL-6 and TNF- $\alpha$ levels for secondary glaucoma. ROC curves indicating the diagnostic values of aqueous humoral IL-17, IL- 6 and TNF- $\alpha$ levels for secondary glaucoma are shown in Fig. 2. The area 

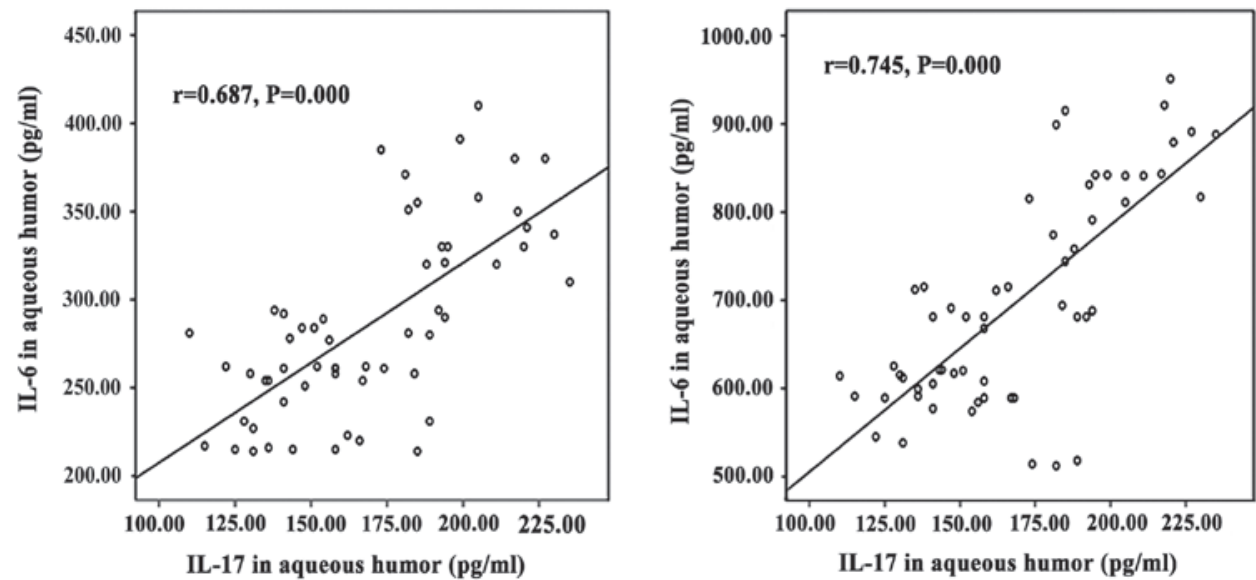

Figure 1. Correlation analysis of IL-17 levels with IL-6 and TNF- $\alpha$ levels. IL-17 levels in the aqueous humor was positively correlated to IL-6 and TNF- $\alpha$ levels.

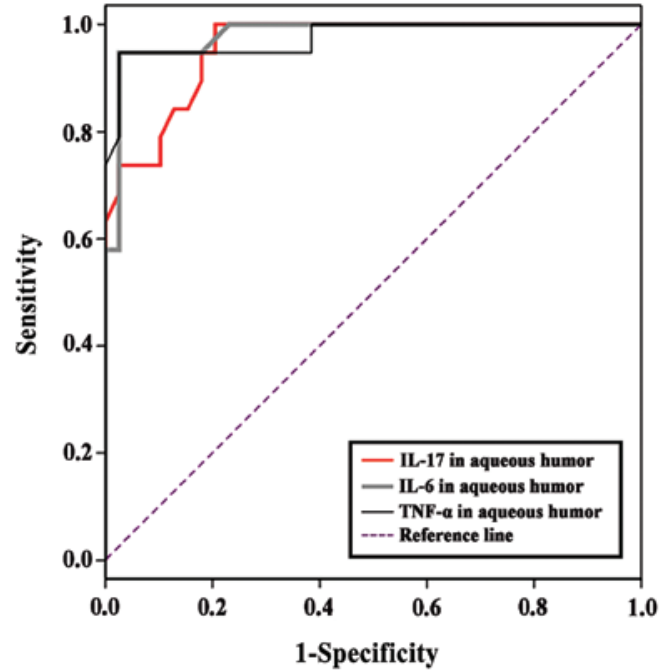

Figure 2. ROC curves showing the diagnostic value of aqueous humoral IL-17, IL- 6 and TNF- $\alpha$ levels for secondary glaucoma. The large areas under the curve showed that aqueous humor levels of IL-17, IL- 6 and TNF- $\alpha$ could be applied for the diagnosis of secondary glaucoma.

under the curve for IL-17 for the prediction of glaucoma was 0.957 ( $\beta=0.023, \mathrm{P}<0.001,95 \%$ CI, 0.913-1.000). The optimal cut-off value for the diagnosis of secondary glaucoma was $177.5 \mathrm{pg} / \mathrm{ml}$ with a sensitivity of 1.000 and a specificity of 0.881 . The area under the curve for IL- 6 for the prediction of glaucoma was 0.980 ( $\beta=0.016, \mathrm{P}<0.001,95 \% \mathrm{CI}, 0.949-1.000)$. The optimal cut-off value for the diagnosis of secondary glaucoma was $302.0 \mathrm{pg} / \mathrm{ml}$ with a sensitivity of 0.947 and a specificity of 0.974 . The area under the curve for TNF- $\alpha$ for the prediction of glaucoma was $0.975(\beta=0.021, \mathrm{P}<0.001,95 \%$ CI, 0.933-1.000). The optimal cut-off value for the diagnosis of secondary glaucoma was $751.0 \mathrm{pg} / \mathrm{ml}$ with a sensitivity of 0.947 and a specificity of 0.974 .

\section{Discussion}

Vitreoretinal surgery is an important treatment method for retinal detachment, retinal vein occlusion and other diseases $(6,7)$. With the development of medical technology, its safety has been significantly improved, but incidences of injected silicone oil-related complications still exist. Teke et al (8) reported that $10-40 \%$ of patients suffered from secondary glaucoma after surgery. This may be because the biological functions of silicone oil could not completely replace those of the vitreous. Silicone oil filling can potentially impede nutrition exchange between inner vitreous cells and the vitreous. Also, it cuts off effective molecular stimuli to the inner cells. Together with feedback production of harmful substances, these adverse effects of silicon oil can cause glaucoma, as well as serious and permanent damage to the optic nerve cells (9-11). Previous studies have shown that glaucomarelated injuries were associated with ocular hypertension. However, recent studies have shown that serious optic nerve damage can also occur in glaucoma patients without ocular hypertension $(12,13)$, suggesting that it was difficult to predict secondary glaucoma by monitoring IOP alone, and that there were other biological mechanisms causing glaucomatous optic nerve damage. Secondary glaucoma caused by silicone oil tamponade typically showed inconsistences between clinical symptoms and IPO values. The majority of these patients showed no obvious symptoms. Only a small number of patients had mild swelling and blurred vision when the IOP was over $40 \mathrm{mmHg}$. However, physical examination still did not show conjunctival hyperemia and the pupils often lacked characteristic signs, making it difficult to predict glaucoma. By the time the patients first presented obvious symptoms, irreversible damage had often already occurred (14-17). This study showed that the observation group and the control group did not have significant differences regarding incidence of silicone particles in the anterior chamber, peripheral anterior synechiae, pupillary block, silicone oil emulsification, artificial intraocular lens, and aphakia, suggesting the development of other complications did not increase the risk of secondary glaucoma. This situation made the early identification and control of secondary glaucoma more difficult.

Inflammation is one of the mechanisms by which glaucoma causes damage to the optic nerve cells. Ohira et al (18) observed that concentrations of IL-6, IL- 8 and TNF- $\alpha$ in the aqueous humor were 171.1, 214.5 and $3.5 \mathrm{pg} / \mathrm{ml}$, respectively, in patients with uveitic glaucoma. These numbers were signifi- 
cantly higher than those found in people without glaucoma. Yi et al (19) found elevated levels of IL-6 and VEGF in the serum and aqueous humor of patients with neovascular glaucoma. In addition, they noted that ranibizumab could treat neovascular glaucoma by inhibiting the expression of IL-6 and VEGF. Based on these findings, we speculated that inflammation was also involved in the pathogenesis of secondary glaucoma after silicone oil tamponade. We examined the expression levels of IL-17 and its effector molecules IL-6 and TNF- $\alpha$ in aqueous humor samples. The results showed that patients with secondary glaucoma had IL-17 levels of $204.2 \pm 18.3 \mathrm{pg} / \mathrm{ml}$, which was significantly higher than in those without secondary glaucoma $(152.3 \pm 22.2 \mathrm{pg} / \mathrm{ml})$, suggesting IL-17 might be involved in the development of secondary glaucoma after silicone oil tamponade IL-17 is an important inflammatory mediator involved in the pathogenesis of many diseases, and is the initial element in inflammatory responses (20). The increased IL-17 in the aqueous humor of patients with silicone oil tamponade could promote the aggregation of neutrophils and macrophages, which would lead to inflammation (21). Neutrophils and macrophages could worsen inflammation primarily by secreting IL-6, TNF- $\alpha$ and other factors (22). TNF- $\alpha$ is directly involved in induction of apoptosis and could destroy the function of the trabecular cells (23). IL-6 is a pleiotropic factor which can induce the expression of a variety of inflammatory mediators and the activation of lymphocytes and NK cells, thus, exacerbating inflammation (24). This study showed that the expression of IL-17 was positively correlated with that of IL- 6 and TNF- $\alpha$, confirming that IL-17 could induce IL-6 and TNF- $\alpha$. Also, these results indicated that inflammation was involved in the optic nerve damage. Understanding the upstream regulatory mechanisms of glaucoma pathogenesis could aid the development of targeted treatment plans.

The direct detection of IL-17, IL- 6 and TNF- $\alpha$ levels may also contribute to the ability to diagnose secondary glaucoma with high sensitivity and specificity, suggesting effective control of misdiagnosis and missed diagnosis. This finding was consistent with the conclusion of Qin et al (25). However, it is difficult to prove that abnormal increases in these indicators could help to predict early phase secondary glaucoma and guide targeted interventions. Follow-up studies are needed to answer these questions.

In conclusion, levels of the inflammatory mediators IL-17, IL- 6 and TNF- $\alpha$ were increased in the aqueous humor of patients with silicone oil tamponade-induced secondary glaucoma, suggesting the involvement of inflammation in the pathogenesis of this type of glaucoma. Early monitoring of inflammatory reactions in patients might help to improve the effective control of secondary glaucoma.

\section{References}

1. Jančo L, Tkáčová Villemová K, Ondrejková M, Vida R, BartošM and Mesárošová M: Retinal tamponade with silicone oil - long term results. Cesk Slov Oftalmol 70: 178-182, 2014 (In Czech)

2. Scheerlinck LM, Schellekens PA, Liem AT, Steijns D and Leeuwen R: Incidence, risk factors, and clinical characteristics of unexplained visual loss after intraocular silicon oil for macula-on retinal detachment. Retina 36: 342-350, 2016.
3. Miller JB, Papakostas TD and Vavvas DG: Complications of emulsified silicone oil after retinal detachment repair. Semin Ophthalmol 29: 312-318, 2014.

4. Zhang Z and Luan J: Clinical observations of secondary glaucoma post silicone oil tamponade. Chin J Ocul Fundus Dis 27: 363-365, 2011.

5. Wasilewska A, Winiarska M, Olszewska M and Rudnicka L: Interleukin-17 inhibitors. A new era in treatment of psoriasis and other skin diseases. Postepy Dermatol Alergol 33: 247-252, 2016.

6. Wang F: Efficacy of selective laser trabeculoplasty in the treatment of secondary glaucoma eye after silicone oil tamponade. Chin J Prim Med Pharm 21: 2475-2477, 2014.

7. Dooley IJ, Duignan ES and Kilmartin DJ: Long-term heavy silicone oil intraocular tamponade. Int Ophthalmol 36: 3-7, 2016.

8. Teke MY, Elgin U, Sen E, Ozdal P and Ozturk F: Intravitreal silicone oil induced changes in corneal biomechanics. Int Ophthalmol 34: 457-463, 2014.

9. Kong YL, Hu XR, Gao JH and Zheng LD: Clinical analysis of secondary glaucoma after silicone oil tamponade. Shanxi Med J 42: 1280-1281, 2013.

10. Gao L and Liu S: Anterior chamber silicone oil emulsion. Zhonghua Yan Ke Za Zhi 50: 379-379, 2014 (In Chinese).

11. Rosca C, Munteanu M, Tamasoi I, Petrovic Z, Balica N, Nicula C and Cretu O: Calcification of hydrophilic acrylic intraocular lens in eyes with silicone oil tamponade - an interventional case series report. Acta Ophthalmol 94: 625-627, 2016.

12. Ichhpujani P, Jindal A and Jay Katz L: Silicone oil induced glaucoma: A review. Graefes Arch Clin Exp Ophthalmol 247: 1585-1593, 2009.

13. Farrahi F, Feghhi M, Ostadian F and Alivand A: Pars plana vitrectomy and silicone oil injection in phakic and pseudophakic eyes; corneal endothelial changes. J Ophthalmic Vis Res 9: 310-313, 2014.

14. Falavarjani KG, Modarres M and Nazari H: Therapeutic effect of bevacizumab injected into the silicone oil in eyes with neovascular glaucoma after vitrectomy for advanced diabetic retinopathy. Eye (Lond) 24: 717-719, 2010.

15. Zoric Geber M, Bencic G, Vatavuk Z, Ivekovic R and Friberg TR: Retinal nerve fibre layer thickness measurements after successful retinal detachment repair with silicone oil endotamponade. Br J Ophthalmol 99: 853-858, 2015.

16. Guo XQ, Tian B, Liu ZC, Wei WB, Tao Y, Sun SJ and Zhang Y: A new rat model of glaucoma induced by intracameral injection of silicone oil and electrocoagulation of limbal vessels. Chin Med J (Engl) 124: 309-314, 2011.

17. Zhang M, Li B, Wang J, Liu W, Sun Y and Wu X: Clinical results of selective laser trabeculoplasty in silicone oil-induced secondary glaucoma. Graefes Arch Clin Exp Ophthalmol 252: 983-987, 2014.

18. Ohira S, Inoue T, Iwao K, Takahashi E and Tanihara H: Factors influencing aqueous proinflammatory cytokines and growth factors in uveitic glaucoma. PLoS One 11: e0147080, 2016.

19. Yi Z, Jiang S, Liu T and Du EG: Effects of ranibizumab on the serum and aqueous humor IL- 6 and VEGF in patients with neovascular glaucoma. Chin J Biol Pharm 36: 151-153, 2016.

20. Guedes MC, Borrego LM and Proença RD: Roles of interleukin-17 in uveitis. Indian J Ophthalmol 64: 628-634, 2016.

21. Speeckaert R, Lambert J, Grine L, Van Gele M, De Schepper S and van Geel N: The many faces of interleukin-17 in inflammatory skin diseases. Br J Dermatol 175: 892-901, 2016.

22. Boshtam M, Asgary S, Kouhpayeh S, Shariati L and Khanahmad H: Aptamers against pro- and anti-inflammatory cytokines: A review. Inflammation 40: 340-349, 2016.

23. Bećarević M: TNF-alpha and annexin A2: Inflammation in thrombotic primary antiphospholipid syndrome. Rheumatol Int 36: 1649-1656, 2016

24. Kumari N, Dwarakanath BS, Das A and Bhatt AN: Role of interleukin-6 in cancer progression and therapeutic resistance. Tumour Biol 37: 11553-11572, 2016.

25. Qin Y, Chen X, Liu J and Dai SY: The expression of aqueous humor IL-17 in patients with secondary glaucoma caused by silicon oil tamponade and its relationship with glaucoma. Chin J Ocul Trauma Occup Eye Dis 38: 365-368, 2016. 